\title{
ARTÍCULOS
}

Sometido 13.05.2015. Aprobado 23.12.2015

Evaluado por el sistema double blind review. Editor Científico: Jorge Verschoore

DOI: http://dx.doi.org/10.1590/Soo34-759020160208

\section{PROPENSIÓN A INNOVAR EN TIY RECIPROCIDAD EN EL ÁMBITO DE LAS REDES SOCIALES}

\author{
Propensão a inovar em $\mathrm{Tl}$ e reciprocidade no âmbito das redes sociais \\ Personal innovativeness in IT and reciprocity in the domain of social \\ networks
}

\begin{abstract}
RESUMEN
El objetivo de la investigación reside en la influencia de la propensión a la innovación en Tecnologías de la Información (TI) como rasgo personal específico, y la reciprocidad sobre el apoyo recibido (su percepción) y la integración social en las redes sociales online. La revisión de la literatura revela la ausencia de estudios concluyentes sobre los efectos de las diferencias personales en la integración social. Un modelo estructural se propone para valorar las relaciones entre los constructos, y el poder predictivo del modelo de investigación. Los resultados demuestran que mejorar la reciprocidad, el apoyo recibido y la promoción de la propensión a la innovación en TI son iniciativas relevantes para el bienestar del usuario y su integración social. La investigación amplía el poder explicativo de la teoría existente, y sugiere a los responsables de marketing estrategias que mejoren dichas variables fomentando la integración social y el éxito duradero de la red social online.
\end{abstract}

PALABRAS CLAVE | Redes sociales online, reciprocidad, propensión a la innovación en tecnologías de la información, integración social, apoyo comunitario percibido.

\section{RESUMO}

O objetivo deste estudo é investigar a influência da propensão a inovar em Tecnologias da Informação (TI) como um traço específico e os efeitos da reciprocidade no apoio recebido (sua percepção) e integração social nas redes sociais. A revisão de literatura revela a ausência de estudos conclusivos sobre os efeitos das diferentes características pessoais na integração social. Um modelo estrutural propõe estabelecer em duas etapas as relações entre os constructos e o poder preditivo para avaliar o modelo de pesquisa pretendido. Os resultados demonstram que o aumento da reciprocidade, o apoio recebido, e a promoção da propensão a inovar em TI são iniciativas relevantes que promovem o bem-estar dos usuários e sua integração social. Esta pesquisa, portanto, melhora o poder explicativo da teoria existente e sugere estratégias que melhorem a integração social e o sucesso das redes sociais.

PALAVRAS-CHAVE / Redes sociais, reciprocidade, propensão a inovar em Tecnologia da Informação, integração social, apoio social percebido.

\section{MIRIAN MUÑOZ-EXPOSITO \\ mirian@us.es \\ Profesora de la Universidad de Sevilla, Facultad de Ciencias \\ Económicas y Empresariales - Sevilla, España}

\section{MANUEL JESUS SÁNCHEZ-FRANCO} majesus@us.es

Profesor de la Universidad de Sevilla, Facultad de Ciencias Económicas y Empresariales - Sevilla, España

\begin{abstract}
The objective of this study is to investigate the influence of personal innovativeness in Information Technologies (IT) as a specific trait and the effects of reciprocity on community support (their perception) and social integration in the domain of social network site innovation. A review of the literature reveals that scarce studies analyze the effects of personal differences on social integration. Structural equation modelling is proposed to assess the relationships between the constructs, along with the predictive power of the research model. The results demonstrate that increasing reciprocity, community support, and assisting members' personal innovativeness are relevant initiatives that promote users' wellbeing, and their social integration. This research therefore improves the explanatory power of existing theoretical frameworks, and suggests to marketers several strategies that improve the social integration and enduring success of social network site.
\end{abstract}

KEYWORDS / Social Network Sites, reciprocity, personal innovativeness in Information Technologies, social integration, social perceived support. 


\section{INTRODUCCIÓN}

Los medios sociales online se basan en el intercambio sostenido de información y conocimiento generados por el usuario, y se conciben como recursos de búsqueda y consumo de información esenciales (Harris \& Dennis, 2011). Precisamente el éxito de una red social deriva de su sostenibilidad a largo plazo; las relaciones deben ser duraderas entre los miembros que comparten experiencias y conocimientos. No obstante, los medios sociales online evidencian escaso éxito en la retención de sus miembros (Wasko \& Faraj, 2005), originando un debate sobre los antecedentes de la participación del usuario y su integración social.

En general, los usuarios no interactúan en entornos vulnerables (Gefen, 2000), siendo el entorno online un entorno complejo debido a la falta de contacto físico que inhibe la participación, el apoyo y la integración comunitaria del usuario. En este contexto, nuestro estudio analiza factores reductores de la incertidumbre y consecuentemente, promotores del uso regular de los entornos sociales - más allá de los antecedentes ya abordados en los modelos de aceptación tecnológica (Hester, 2011). Se analizan la reciprocidad como norma social beneficiosa que aporta tranquilidad a los miembros ante la expectativa de que sus aportaciones a la comunidad les serán devueltas de alguna forma en el futuro, y la propensión a la innovación en Tecnologías de la Información (TI) (cf. personal innovativeness in the domain of information technology; en adelante, PIIT; Agarwal \& Prasad, 1998) como una característica personal que ayuda a identificar individuos propensos a adoptar innovaciones de TI (Rogers, 2003, p. 280). Ambos factores impulsan el enriquecimiento de relaciones mutuamente beneficiosas basadas en el apoyo comunitario percibido (Herrero \& Gracia, 2007).

Si bien la falta de referencias sociales como el lenguaje corporal, la concurrencia de usuarios con diferentes costumbres y perspectivas sociales y la falta de inmediatez en el entorno online, generan holgazanería social (Ma \& Agarwall, 2007), el conocimiento mutuo y un deseo de colaboración enfatizados por la presión normativa derivada de la reciprocidad predisponen a una mayor contribución debido a la percepción de la probable devolución del esfuerzo colaborativo previo (Kankanhalli, Tan, \& Wei, 2005). La reciprocidad fomenta actividades de colaboración mutua en entornos sociales online, y consecuentemente estabiliza las relaciones creadas (Nahapiet \& Ghoshal, 1998; Putnam, 1995). Asimismo, los usuarios con mayor PIIT consideran las redes sociales útiles y fáciles de usar, toleran un mayor nivel de incertidumbre y dependen menos de la opinión subjetiva de otros (Rogers, 1995). Más aun, comunican su identidad de manera efectiva y fomentan sus relaciones en la comunidad (Chou, 2010), y fortalecen sus conductas sociales al relativizar parcialmente sus riesgos. El constructo PIIT complementa pues los modelos de aceptación y uso de las TI, al impulsar conductas prosociales e intensificar las relaciones a través de la interacción mutua evidenciada en el apoyo comunitario percibido -considerado en esta investigación la clave esencial para el éxito sostenido de las redes sociales (Brodie, Ilic, Juric, \& Hollebeek, 2013; Rothaermel \& Sugiyama, 2001).

En las secciones siguientes, nuestro trabajo define los componentes del apoyo comunitario percibido, y conceptualiza la reciprocidad y PIIT como claves predictoras. Se construye un modelo de hipótesis y se testa empíricamente. Se diseñan las consideraciones finales e implicaciones gerenciales así como futuras líneas y limitaciones de la investigación.

\section{APOYO COMUNITARIO PERCIBIDO}

El apoyo comunitario percibido es un indicador esencial del éxito de la comunidad debido a su papel como predictor de la adaptación de los individuos a eventos estresantes de su vida (Wethington \& Kessler, 1986). Es considerado una fuente potencial de apoyo y participación en la red social, descomponiéndose en apoyo recibido, participación e integración comunitaria (Herrero \& Gracia, 2007).

Por un lado, el apoyo recibido se define como el conjunto de recursos afectivos disponibles en la red (i.e. las provisiones subjetivas proporcionadas por la red). Por otro lado, la integración social, reflejada en la participación e integración comunitaria (Gracia, García, \& Musitu, 1995), favorece el desarrollo comunitario y la capacidad de las comunidades para activar recursos humanos internos, resolver problemas que afectan a los individuos y promover el empoderamiento social. La participación se asocia con la implicación en el entramado social que abarca las acciones en comunidad; e.g., obtener y compartir conocimiento o experiencias relativo a un interés común (Casaló, Flavián, \& Guinalíu, 2007). La integración comunitaria se identifica con el sentimiento subjetivo de vinculación, pertenencia e identidad individual y social (Tonteri, Kosonen, Ellonen, \& Tarkiainen, 2011).

Diversos estudios manifiestan que la cantidad y profundidad del apoyo se relacionan positivamente con la participación de sus miembros (Algesheimer, Dholakia, \& Herrmann, 2005; Tonteri et al., 2011). El usuario muestra mayor grado de contribución social si percibe muestras de apoyo de su comunidad -fortaleciendo con ello la creencia de continuar obteniendo beneficios emocionales de las relaciones creadas, y deseando continuar con una relación gratificante. La recepción de apoyo es pues esencial para el 
desarrollo de la integración social (Sánchez-Franco, BuitragoEsquinas, \& Hernández-Mogollón, 2012).

Se formula la siguiente hipótesis:

$\mathrm{H} 1$ : El apoyo recibido influye positivamente en la integración social.

\section{COMPORTAMIENTO PROSOCIAL Y NORMA DE RECIPROCIDAD}

En general, las redes sociales promueven comportamientos prosociales, i.e. conductas con consecuencias sociales positivas y efectos beneficiosos (e.g., comportamientos de ayuda, apoyo, comprensión, cooperación y empatía) (Osorio, 2009). Como señala Vallor (2012), los medios sociales impulsan a los individuos a realizar acciones nobles, rigiéndose la norma de reciprocidad por principios basados en el intercambio - tal que los esfuerzos de compartir experiencias y conocimientos con otros se recompensen. El orden social se establece por un "sistema de prestaciones recíprocas generador de una dependencia mutua entre las partes, asegurando el cumplimiento de las normas" (Gonnet, 2010, p. 2). La reciprocidad se concibe precisamente como la red de entendimientos mutuos, obligaciones y expectativas de comportamiento por los demás. En este sentido, a mayor nivel de reciprocidad el individuo interactúa y participa más activamente (Wasko \& Faraj, 2005), promoviendo el desarrollo de apoyo comunitario percibido. Como manifiestan Wang y Fesenmaier (2003), la reciprocidad es un motivo principal que impulsa la contribución y participación de los individuos en comunidades online, promueve la adhesión de los miembros a las normas, reglas y valores de la comunidad, incrementa el vínculo con la misma e impulsa los sentimientos de pertenencia e identidad (Blanchard, 2008).

Se formula la siguiente hipótesis:

H2: La reciprocidad influye positivamente en la integración social.

Más aún, el apoyo recibido incrementa la probabilidad de ejecutar comportamientos prosociales basados en comportamientos previos de otros miembros (Ruiz, 2005), creándose un sentimiento informal de solidaridad entre ellos y fomentándose la reciprocidad (Cothrel \& Williams, 1999). Se formula la siguiente hipótesis:

H3: El apoyo recibido influye positivamente en la reciprocidad.

Asimismo, el individuo contribuye devolviendo la ayuda recibida, y experimentando un sentido de pertenencia e identidad con el grupo. Si el usuario no recibe apoyo, pierde interés por la comunidad, provocando la reducción de su nivel de interacción mutua y debilitándose la integración social. Se formula la siguiente hipótesis:

H4: La relación entre apoyo recibido e integración social está mediada parcial y positivamente por la reciprocidad.

\section{PROPENSIÓN A INNOVAR EN EL ÁMBITO DE LAS TI}

La percepción de una innovación tecnológica (e.g., redes sociales online) se basa en características de la propia tecnología (e.g., calidad del sistema), factores situacionales (e.g., formación de los usuarios o soporte tecnológico) y cualidades del usuario (e.g., autoeficacia o experiencia previa) (Lee, Kozar, \& Larsen, 2003). En particular, las cualidades personales son rasgos influyentes y predictores del comportamiento (Woszczynski, Roth, \& Segars, 2002), y desempeñan un papel clave en la aceptación de las innovaciones en general (Rogers, 2003). La identificación y comprensión de las cualidades del individuo con influencia sobre la decisión de aceptación es esencial, y debe modelizarse en la predicción de uso (Yi, Fiedler, \& Park, 2006, p. 400).

Precisamente, PIIT se concibe como un rasgo individual y estable (Agarwal \& Prasad, 1998) - no influido por variables ambientales o internas. Por un lado, las redes sociales no están exentas de riesgo pese a ser fuente de recursos benéficos (Nowell \& Boyd, 2010). En este contexto, PIIT modera la capacidad de tolerancia al riesgo, promoviendo la aceptación de las redes sociales online (Thatcher \& Perrewe, 2002). Más aún, PIIT correlaciona con la eficacia percibida del individuo, demandando menor necesidad de conocimientos y experiencias relacionadas, siendo pues un determinante directo y significativo de la utilidad y facilidad de uso percibida (Yi et al., 2006), y se relaciona con la participación social (Rogers, 1995). Los individuos propensos a intentar una $\mathrm{TI}$ consideran las redes sociales útiles y sencillas, y tienden a participar asumiendo riesgos y atendiendo las peticiones de ayuda. Asimismo, los usuarios con mayor PIIT manifiestan sentimientos positivos de identidad y de apego a los otros (Chou, 2010). PIIT se considera un antecedente significativo de la sensación placentera asociada con el sentimiento de apoyo. Los individuos con mayor PIIT están pues más predispuestos a un uso adecuado de las herramientas de la comunidad y aportar con ello conocimiento y apoyo eficaz (Thatcher \& Perrewe, 2002); tenderán a ser proactivos en la aceptación del entorno social y la asunción de riesgos, resolverán conflictos y generarán ideas y pensamientos a través de las interacciones online, y se gratificarán sensorial y cognitivamente (Sánchez-Franco, Buitrago-Esquinas, 
\& Yñiguez-Ovando, 2013). Son embajadores de la red social; i.e. ayudan a construir la comunidad reclutando nuevos miembros (Preece, 2000), asumiendo el rol de guía de los comportamientos de otros miembros ofreciendo retroalimentación de apoyo (Gurzick \& Lutters, 2009) e impulsando la reciprocidad. Se fomenta un sentimiento de apoyo a la red social online y también de ser apoyado.

Se formulan las siguientes hipótesis:

H5: La propensión a innovar en el ámbito de las TI, influye positivamente en la integración social;

H6: La propensión a innovar en el ámbito de las TI, influye positivamente en la reciprocidad; y

H7: La propensión a innovar en el ámbito de las TI, influye positivamente en el apoyo recibido.

\section{MÉTODO}

\section{Procedimiento}

Nuestra investigacion pregunta a los encuestados por su pertenencia en las redes sociales online. En caso de pertenencia responden voluntariamente un cuestionario offline relacionado con la red empleada frecuentemente. Por razones de dificultad metodológica, coste, sentido práctico y al tratarse de una población capacitada el muestreo por conveniencia es un método adecuado para validar nuestro modelo (Sue, 2012).

Se revisan las respuestas emitidas para detectar y corregir ambigüedades, respuestas ilegibles, omisiones o incoherencias (Ruiz, Izquierdo, \& Piñera, 1998). En general, las respuestas confusas ocasionan sesgos en las conclusiones, dando lugar a casos atípicos con valores extremos - notablemente diferentes de las restantes observaciones - que contaminan la muestra, afectan negativamente los análisis estadísticos efectuados, y reducen la probabilidad de detectar violaciones sistemáticas de la teoría cuando esta es falsa (Calder, Lynn, \& Tybout, 1981). Su detección (mediante Mahalanobis $D^{2}, \mathrm{p}<0.001$ ) y tratamiento homogeniza la muestra y reduce asimismo las influencias de terceros factores no modelizados. En particular, 27 cuestionarios presentan casos atípicos, ambigüedades o ilegibilidades no subsanables. Se procede a su eliminación manteniendo la utilidad del conjunto de datos para el análisis estadístico y minería de datos, e impidiendo pues que casos influyentes distorsionen los resultados (Osborne \& Overbay, 2008). Los datos han sido recogidos mediante cuestionario autoadministrado anónimamente, no pudiéndose contactar nuevamente con el encuestado (Ruiz et al., 1998). En suma, la muestra final consta de 492 cuestionarios.

\section{Participantes}

La población universitaria es un importante segmento de usuarios de Internet (McKnight, Kacmar, \& Choudhury, 2004). Los adolescentes y los jóvenes o adultos emergentes universitarios son consumidores relevantes de esta tecnología en comparación con la población general y la emplean para comunicarse con sus iguales (Boneva, Quinn, Kraut, Kiesler, \& Shklovski, 2006). La adultez emergente se define como un estadio de exploracion e inestabilidad (Arnett, 2004, p. 21), que define su identidad e intimidad a través de interconexiones con parejas, amigos y familiares (Subrahmanyam, Reichc, Waechterb, \& Espinoza, 2008). La investigación descriptiva y etnográfica sobre el comportamiento de los jóvenes en Internet y los medios sociales evidencian que las redes sociales son esenciales para el desarrollo de la identidad personal, median en las relaciones de los jóvenes con sus amigos, parejas y grupos amplios (Agosto \& Abbas, 2010; Ito et al., 2009) e impulsan el apoyo emocional, la confianza y la lealtad (Radmacher \& Azmitia, 2006) - fomentando la obtención de apoyo social (Larose, Eastin, \& Gregg, 2001). Investigaciones previas describen el uso de las redes sociales entre los estudiantes universitarios y su rol como participantes activos (García, Alonso, \& Hoyo, 2013; entre otros) -promoviendo en ellas la interacción social y reciprocidad no sólo para alcanzar metas académicas sino también fines de comunicación, disfrute y sociabilización (Leung \& Lee, 2012; Osorio, Molero, Pérez, \& Mercader, 2014). En la muestra analizada un 21,9\% de los usuarios mantiene vínculos con más de 150 contactos, y el 43,2\% con más de 200 contactos, constituyéndose en impulsores de la reciprocidad. Más aún, el uso de las redes se ha convertido en una actividad cotidiana (Gómez, Roses, \& Farías, 2012). El $61 \%$ de los encuestados accede a ellas más de una vez al día. El $52,5 \%$ es usuario de una o varias redes sociales desde hace mas de dos años. Los usuarios mas participativos son los estudiantes 92,0\% (INE, 2014) y jovenes de 16 a 24 años son 91,3\% (INE, 2014). En suma, los estudiantes universitarios constituyen el grupo mas propenso a compartir informacion y opiniones, participar en eventos, apoyarse mutuamente, desarrollar su identidad, $u$ organizar grupos (Ahn, 2011, 2012; OBS, 2014), conformando un segmento de relevancia para el estudio de PIIT, la reciprocidad y la integracion social.

En nuestra investigación los valores de sexo y edad se encuentran en consonancia con la distribución de audiencias y comportamientos en este medio social. La edad media de los encuestados es igual a 21,3 años (desviación típica: 2,4 años). El $61,8 \%$ son mujeres. Las redes sociales mas utilizadas (i.e. Facebook, $53,3 \%$, y Twitter, 46,3\%) se corresponden con las tres redes sociales online mas empleadas en Espana (IAB, 2015) (cf. Tabla 1, 2 у 3). 
Tabla 1. Características sociodemográficas de la muestra

\begin{tabular}{|c|c|}
\hline Variables & Perfil \\
\hline Sexo $^{\text {a }}$ & $\%$ \\
\hline Hombre & 37,8 \\
\hline Mujer & 61,8 \\
\hline Edad (años) ${ }^{b}$ & $\%$ \\
\hline Media & 21,3 \\
\hline Desviación estándar & 2,4 \\
\hline Frecuencia de uso & $\%$ \\
\hline Menos de 1 vez a la semana & 1,2 \\
\hline Al menos 1 vez a la semana & 3,0 \\
\hline 203 veces a la semana & 4,1 \\
\hline Varias veces por semana & 8,3 \\
\hline Al menos una vez al día & 22,4 \\
\hline Varias veces al día & 61,0 \\
\hline Red social (más frecuente) & $\%$ \\
\hline Facebook & 53,3 \\
\hline Twitter & 46,3 \\
\hline Otras & 0,4 \\
\hline Tiempo de membresía ${ }^{c}$ & $\%$ \\
\hline Menos de un año & 4,7 \\
\hline Más de un año & 24,8 \\
\hline Más de dos años & 52,5 \\
\hline Más de cinco años & 17,3 \\
\hline Más de ocho años & 0,6 \\
\hline Cantidad de contactos $^{d}$ & $\%$ \\
\hline Menos de 50 & 2,9 \\
\hline Entre 50 y 100 & 12,7 \\
\hline Entre 101 y 150 & 19,3 \\
\hline Entre 151 y 200 & 21,9 \\
\hline Más de 200 & 43,2 \\
\hline Número total de encuestados & 492 \\
\hline
\end{tabular}

${ }^{\mathrm{a}} 0,4 \%$ de datos perdidos, ${ }^{\mathrm{b}} \mathrm{0}, 6 \%$ de datos perdidos. ${ }^{\mathrm{c}} \mathrm{0}, 2 \%$ de datos perdidos. do, 8 de datos perdidos.
Tabla 2. Segmento de usuarios de Facebook por sexo y edad

\begin{tabular}{l|c|c|c|c|c}
\hline Facebook & \multicolumn{5}{|c}{ Usuarios por sexo y edad } \\
\hline $\begin{array}{l}\text { Rangos de } \\
\text { Edad }\end{array}$ & Hombres & $\begin{array}{c}\% \\
\text { válido }\end{array}$ & Mujeres & $\begin{array}{c}\% \\
\text { válido }\end{array}$ & $\begin{array}{c}\% \text { Total } \\
\text { válido }\end{array}$ \\
\hline $18-30$ años & 78 & 29,89 & 178 & 68,2 & 98,09 \\
\hline $\begin{array}{l}\text { Más de 31 } \\
\text { años }\end{array}$ & 2 & 0,76 & 3 & 1,15 & 1.91 \\
\hline $\begin{array}{l}\text { Total } \\
\text { encuestados }\end{array}$ & 80 & 30,65 & 181 & 69,35 & 100 \\
\hline $\begin{array}{l}\text { Total muestra } \\
\begin{array}{l}\text { Total } \\
\text { cuestionarios } \\
\text { válidos }\end{array}\end{array}$ \\
\hline
\end{tabular}

Tabla 3. Segmento de usuarios de Twitter por sexo y edad

\begin{tabular}{l|c|c|c|c|c}
\hline Twitter & \multicolumn{5}{|c}{ Usuarios por sexo y edad } \\
\hline $\begin{array}{l}\text { Rangos de } \\
\text { Edad }\end{array}$ & Hombres & $\begin{array}{c}\% \\
\text { válido }\end{array}$ & Mujeres & $\begin{array}{c}\% \\
\text { válido }\end{array}$ & $\begin{array}{c}\% \text { Total } \\
\text { válido }\end{array}$ \\
\hline $18-30$ años & 106 & 46,7 & 121 & 53,30 & 100 \\
\hline $\begin{array}{l}\text { Más de } 31 \\
\text { años }\end{array}$ & 0 & 0 & 0 & 0 & 0 \\
\hline $\begin{array}{l}\text { Total } \\
\text { encuestados }\end{array}$ & 106 & 46,7 & 121 & 53,30 & 100 \\
\hline \begin{tabular}{l} 
Total muestra \\
\hline $\begin{array}{l}\text { Total } \\
\text { cuestionarios } \\
\text { válidos }\end{array}$
\end{tabular}
\end{tabular}

\section{Análisis de datos}

Se diseña un modelo de ecuaciones lineales estructurales para establecer las relaciones entre constructos. Se estima el poder predictivo del modelo mediante la técnica Partial Least Squares (PLS) (Chin, 1998a, 1998b). El procesamiento de los datos se ejecuta con SmartPLS 2.0, M3. El modelo propuesto se analiza e interpreta en dos etapas, estimando (1) los niveles de fiabilidad y validez convergente y discriminante del modelo de medida, $y$ (2) el modelo estructural.

\section{Medidas}

Se diseña un cuestionario autoadministrado revisándose la literatura para identificar medidas válidas. El contenido de las escalas es adaptado al contexto de las redes sociales. Véase Tabla 4. 
Tabla 4. Modelo de medida - Modelo de primer orden

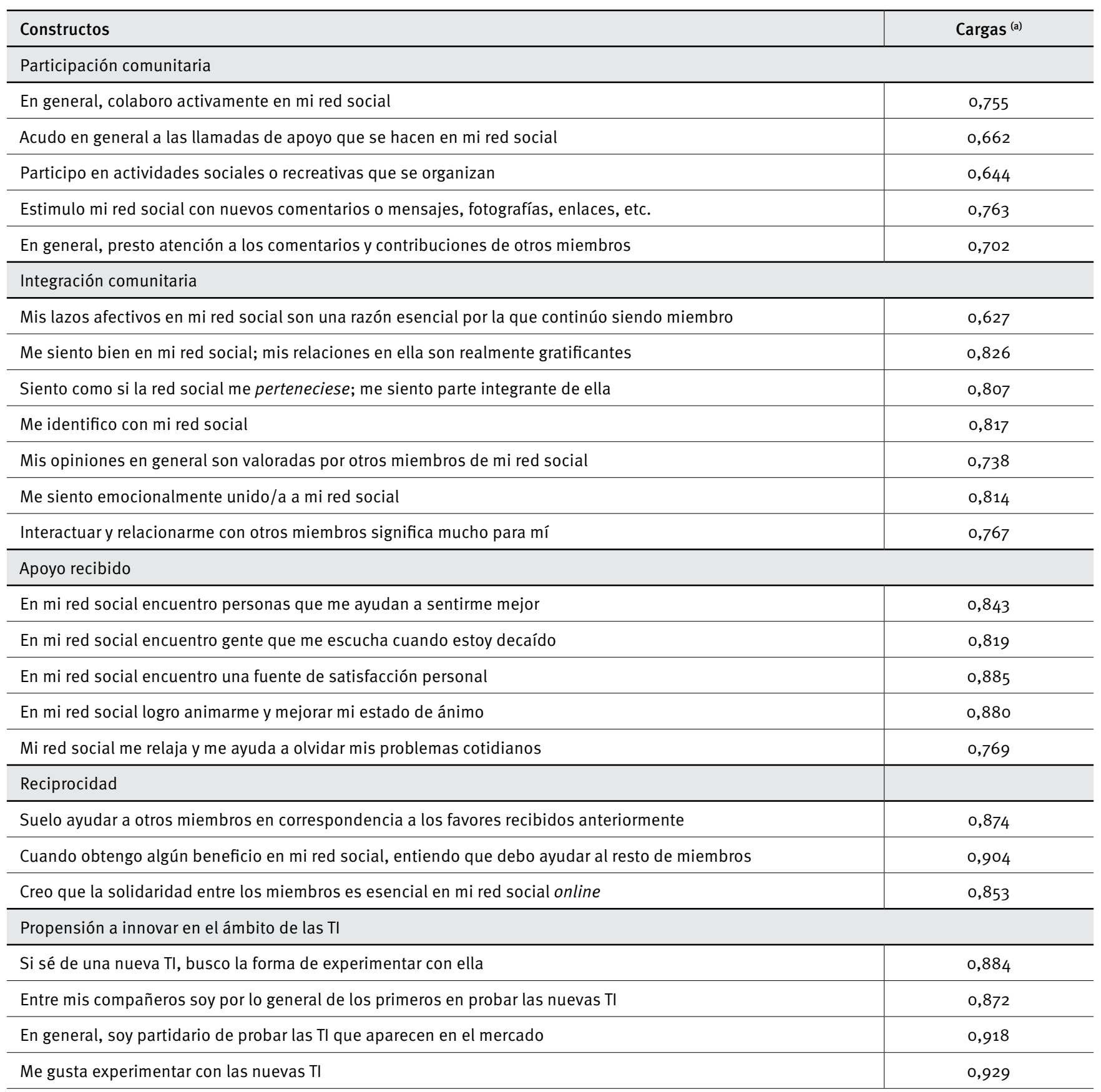

Note: ${ }^{(a)} p<0,001$, basado en $t$ (4.999), dos colas.

Las escalas de medida de las normas de reciprocidad se adaptan de Casaló, Flavián y Guinalíu (2011) y Wiertz y Ruyter (2007) y PIIT, de Agarwal y Prasad (1998). Las dimensiones de apoyo comunitario percibido son adaptadas de Casaló et al. (2007), Herrero y Gracia (2007), entre otros. Un pretest evalúa la idoneidad del texto, el formato y el grado en que mide los constructos. La escala de medición es Likert: 1, totalmente en desacuerdo, a 7, totalmente de acuerdo.

\section{RESULTADOS}

\section{Modelo de medida: Modelo de primer orden}

La fiabilidad individual es valorada examinando las cargas factoriales de los indicadores con su constructo. El indicador debe evidenciar una carga factorial superior a 0,7. En nuestra investigación las cargas factoriales son superiores a 0,7 excepto tres de ellas superiores a 0,6. Investigaciones previas 
recomiendan que el punto crítico se sitúe en o,6 en estudios de tipo exploratorio o cuando las escalas se aplican en contextos diversos (Barclay, Thompson, \& Higgins, 1995; Chin, 1998b). En nuestra investigación incluimos pues cargas superiores a 0,6 al tratarse de escalas de medida validadas en investigaciones previas y asumiendo su carácter exploratorio. Se comprueba la ausencia de perjuicio en la fiabilidad y validez del modelo.

Se comprueba la significación de las cargas factoriales con el procedimiento de remuestreo (bootstrap, 5.000 submuestras) para obtener los estadísticos $t$. Las cargas factoriales son significativas. Véase Tabla 4. La fiabilidad de un constructo se estima a partir del criterio de fiabilidad compuesta $\left(\rho_{c}\right)$ (Fornell \& Larcker, 1981). Nunnally (1978) sugiere el valor o,7 como nivel adecuado de fiabilidad. Los constructos latentes son fiables. Véase Tabla 5.

Tabla 5. Fiabilidad compuesta (modelo de primer orden)

\begin{tabular}{l|c}
\hline Constructos & Fiabilidad compuesta $\left(\mathbf{p}_{\mathrm{c}}\right)$ \\
\hline Apoyo recibido & 0,923 \\
\hline Integración comunitaria & 0,912 \\
\hline Participación comunitaria & 0,832 \\
\hline $\begin{array}{l}\text { Propensión a innovar en el ámbito } \\
\text { de las TI }\end{array}$ & 0,945 \\
\hline Reciprocidad & 0,909 \\
\hline
\end{tabular}

La validez convergente se estima mediante la varianza media extraída (Average Variance Extracted, AVE) (Fornell \& Larcker, 1981). Los valores AVE deben ser superiores (o iguales) a 0,5 . Las varianzas medias extraídas de los constructos cumplen el criterio. Véase Tabla 6.

Tabla 6. Validez convergente (modelo de primer orden)

\begin{tabular}{l|c}
\hline Constructos & AVE \\
\hline Apoyo recibido & 0,707 \\
\hline Integración comunitaria & 0,599 \\
\hline Participación comunitaria & 0,500 \\
\hline $\begin{array}{l}\text { Propensión a innovar en el ámbito } \\
\text { de las TI }\end{array}$ & 0,813 \\
\hline Reciprocidad & 0,770 \\
\hline
\end{tabular}

Finalmente, cada elemento de la diagonal principal (raíz cuadrada del AVE) debe ser superior a los restantes elementos de su fila y columna correspondiente. Los valores fuera de la diagonal representan las correlaciones entre constructos (Barclay et al., 1995). Se acepta la validez discriminante. Véase Tabla 7.

Tabla 7. Validez discriminante (modelo de primer orden)

\begin{tabular}{|c|c|c|c|c|c|}
\hline Constructos & $\begin{array}{c}\text { Apoyo } \\
\text { recibido }\end{array}$ & $\begin{array}{l}\text { Integración } \\
\text { comunitaria }\end{array}$ & $\begin{array}{c}\text { Participación } \\
\text { comunitaria }\end{array}$ & $\begin{array}{c}\text { Propension a } \\
\text { innovar }\end{array}$ & Reciprocidad \\
\hline Apoyo recibido & 0,840 & & & & \\
\hline Integración comunitaria & 0,684 & 0,774 & & & \\
\hline Participación comunitaria & 0,538 & 0,610 & 0,707 & & \\
\hline Propensión a innovar en el ámbito de las TI & 0,253 & 0,357 & 0,362 & 0,901 & \\
\hline Reciprocidad & 0,626 & 0,635 & 0,533 & 0,310 & 0,877 \\
\hline
\end{tabular}

Nota: Cada elemento de la diagonal principal (en negrita) representa la raíz cuadrada del AVE

A continuación, se evalúa la fiabilidad y validez del modelo de medida de segundo orden.

\section{Modelo de medida: Modelo de segundo orden}

El constructo integración social es de segundo orden - medido por los componentes participación e integración comunitaria. En primer lugar, se estima el modelo a partir de los indicadores de los constructos de primer orden (cf. subepígrafe anterior). En segundo lugar, las puntuaciones factoriales se emplean como indicadores del constructo de segundo orden (Chin, 2000).

Los indicadores reflectivos muestran cargas factoriales por encima de 0,7 , y son significativas (véase Tabla 8). Los constructos latentes presentan medidas de consistencia interna que exceden el valor 0,7 (véase Tabla 9). Los valores AVE de los constructos latentes superan el valor 0,5 (véase Tabla 10). Las correlaciones entre constructos son inferiores a la raíz cuadrada del AVE correspondiente (véase Tabla 11). Se acepta la validez convergente y discriminante. 
Tabla 8. Cargas factoriales - Modelo de medida de segundo orden

\begin{tabular}{|c|c|}
\hline Constructos & Cargas ${ }^{(a)}$ \\
\hline \multicolumn{2}{|l|}{ Integración social } \\
\hline Participación comunitaria & 0,876 \\
\hline Integración comunitaria & 0,916 \\
\hline \multicolumn{2}{|l|}{ Apoyo recibido } \\
\hline $\begin{array}{l}\text { En mi red social encuentro personas que me ayudan a } \\
\text { sentirme mejor }\end{array}$ & 0,844 \\
\hline $\begin{array}{l}\text { En mi red social encuentro gente que me escucha } \\
\text { cuando estoy decaído }\end{array}$ & 0,820 \\
\hline $\begin{array}{l}\text { En mi red social encuentro una fuente de satisfacción } \\
\text { personal }\end{array}$ & 0,885 \\
\hline $\begin{array}{l}\text { En mi red social logro animarme y mejorar mi estado de } \\
\text { ánimo }\end{array}$ & 0,879 \\
\hline $\begin{array}{l}\text { Mi red social me relaja y me ayuda a olvidar mis } \\
\text { problemas cotidianos }\end{array}$ & 0,769 \\
\hline Reciprocidad & \\
\hline $\begin{array}{l}\text { Suelo ayudar a otros miembros en correspondencia a los } \\
\text { favores recibidos anteriormente }\end{array}$ & 0,874 \\
\hline $\begin{array}{l}\text { Cuando obtengo algún beneficio en mi red social, } \\
\text { entiendo que debo ayudar al resto de miembros }\end{array}$ & 0,904 \\
\hline $\begin{array}{l}\text { Creo que la solidaridad entre los miembros es esencial } \\
\text { en mi red social online }\end{array}$ & 0,853 \\
\hline Propensión a innovar en el ámbito de las TI & \\
\hline $\begin{array}{l}\text { Si sé de una nueva TI, busco la forma de experimentar } \\
\text { con ella }\end{array}$ & 0,884 \\
\hline $\begin{array}{l}\text { Entre mis compañeros soy por lo general de los primeros } \\
\text { en probar las nuevas } \mathrm{TI}\end{array}$ & 0,873 \\
\hline $\begin{array}{l}\text { En general, soy partidario de probar las TI que aparecen } \\
\text { en el mercado }\end{array}$ & 0,918 \\
\hline Me gusta experimentar con las nuevas TI & 0,929 \\
\hline
\end{tabular}

Nota: $p<0,001$ (basado en t (4.999), dos colas)
Tabla 9. Fiabilidad compuesta (modelo de segundo orden)

\begin{tabular}{l|c}
\hline Constructos & Fiabilidad Compuesta $\left(\mathrm{p}_{\mathrm{c}}\right)$ \\
\hline Integración social & 0,891 \\
\hline Apoyo recibido & 0,923 \\
\hline $\begin{array}{l}\text { Propensión a innovar en el ámbito } \\
\text { de las TI }\end{array}$ & 0,945 \\
\hline Reciprocidad & 0,909 \\
\hline
\end{tabular}

Tabla 10. Validez convergente (modelo de segundo orden)

\begin{tabular}{l|c}
\hline Constructos & AVE \\
\hline Integración social & 0,804 \\
\hline Apoyo recibido & 0,706 \\
\hline $\begin{array}{l}\text { Propensión a innovar en el ámbito } \\
\text { de las TI }\end{array}$ & 0,813 \\
\hline Reciprocidad & 0,770 \\
\hline
\end{tabular}

Tabla 11. Validez discriminante (modelo de segundo orden)

\begin{tabular}{|c|c|c|c|c|}
\hline Constructos & $\begin{array}{c}\text { Apoyo } \\
\text { recibido }\end{array}$ & $\begin{array}{l}\text { Propensión } \\
\text { a innovar en } \\
\text { el ámbito de } \\
\text { las TI }\end{array}$ & Reciprocidad & $\begin{array}{l}\text { Integración } \\
\text { social }\end{array}$ \\
\hline $\begin{array}{l}\text { Apoyo } \\
\text { recibido }\end{array}$ & 0,840 & & & \\
\hline $\begin{array}{l}\text { Propensión } \\
\text { a innovar en } \\
\text { el ámbito de } \\
\text { las TI }\end{array}$ & 0,253 & 0,901 & & \\
\hline Reciprocidad & 0,626 & 0,310 & 0,877 & \\
\hline $\begin{array}{l}\text { Integración } \\
\text { social }\end{array}$ & 0,688 & 0,400 & 0,655 & 0,897 \\
\hline
\end{tabular}

\section{Modelo estructural}

El modelo de investigación explica el 58,8\% de la varianza del constructo integración social y el $41,7 \%$ de la varianza del constructo reciprocidad. Los valores $\mathrm{R}^{2}$ de los constructos dependientes superan el valor o,10 recomendado por Falk y Miller (1992) - a excepción del constructo apoyo recibido. En segundo lugar, la medición de la relevancia predictiva se lleva a cabo mediante el test $\mathrm{Q}^{2}$ (Geisser, 1975). Los valores son superiores a o, aceptando pues la relevancia predictiva. Véase Tabla 12. 
Tabla 12. Poder predictivo $\left(\mathbf{R}^{2}\right)$ y relevancia predictiva $\left(Q^{2}\right)$ del modelo estructural

\begin{tabular}{l|c|c}
\hline Constructos & $\mathbf{R}^{2}$ & $\mathbf{Q}^{2}$ \\
\hline Integración social & 0,588 & 0,455 \\
\hline Apoyo recibido & 0,064 & 0,044 \\
\hline Reciprocidad & 0,417 & 0,311 \\
\hline
\end{tabular}

En tercer lugar, se analiza el tamano del efecto de las variables predictoras de la integracion social (f2) (Chin, 1998b). Cohen (1988) proporciona el siguiente criterio de interpretacion: a) reducido, $0,02<f^{2} \leq 0,15$; b) moderado, $0,15<f^{2} \leq 0,35$, y c) elevado, $f^{2}>0,35$. El apoyo recibido y la reciprocidad muestran los valores 0,284 y 0,143 , respectivamente. El tamaño del efecto de PIIT es 0,072. Una posible explicación reside en la joven edad y el alto nivel educativo de los encuestados. Los usuarios pertenecientes a la muestra son adultos emergentes y universitarios. Un mayor nivel educativo se asocia con una mayor alfabetización tecnológica, y un mayor desarrollo de habilidades instrumentales y cognitivas relacionadas con la información trasmitida a través de TI, y consecuentemente, de la familiaridad (Rothaermel \& Sugiyama, 2001). En general, desarrollan una percepción más favorable y menos riesgosa, y la tendencia pues a aceptar servicios sociales online (Phang et al., 2006).

Tabla 13. Tamaño del efecto, $\mathbf{f}^{2}$

\begin{tabular}{|c|c|c|c|}
\hline & $\mathrm{R}^{2}$ (Int. social) & $\mathrm{R}^{2}$ (Int. social) & \\
\hline Variables predictoras & $\begin{array}{l}\text { Var. pred. } \\
\text { incluida }\end{array}$ & $\begin{array}{l}\text { Var. pred. } \\
\text { excluida }\end{array}$ & $f^{2}$ \\
\hline Reciprocidad & \multirow{3}{*}{0,588} & 0,528 & 0,143 \\
\hline Apoyo recibido & & 0,470 & 0,284 \\
\hline $\begin{array}{l}\text { Propensión a innovar } \\
\text { en el ámbito de las TI }\end{array}$ & & 0,558 & 0,072 \\
\hline
\end{tabular}

En cuarto lugar, siguiendo a Chin (1998a), se ejecuta un bootstrapping con 5.000 casos para generar los errores estándares y los valores $t$ (véase Figura 1). En la Tabla 14 se muestra el contraste de las hipótesis, el efecto de las relaciones, el coeficiente path, y el valor $t$. La variable red social no evidencia efecto interacción significativo sobre las relaciones hipotetizadas.
Tabla 14. Modelo estructural (relaciones directas) Resultados

\begin{tabular}{l|c|c|c|c}
\hline Hipótesis & Signo & $\beta$ & $t$ & Apoyada \\
\hline $\begin{array}{l}\text { H1: Apoyo recibido } \\
\rightarrow \text { Integración social }\end{array}$ & + & $0,438^{*}$ & 11,40 & Sí \\
\hline $\begin{array}{l}\text { H2: Reciprocidad } \\
\rightarrow \text { Integración social }\end{array}$ & + & $0,322^{*}$ & 8,01 & Sí \\
\hline $\begin{array}{l}\text { H3: Apoyo recibido } \\
\rightarrow \text { Reciprocidad }\end{array}$ & + & $0,585^{*}$ & 18,19 & Sí \\
\hline $\begin{array}{l}\text { H5: Propensión a innovar } \\
\text { en el ámbito de las TI } \rightarrow\end{array}$ & + & $0,188^{*}$ & 6,02 & Sí \\
$\begin{array}{l}\text { Integración social } \\
\text { H6: H6: Propensión a innovar } \\
\text { en el ámbito de las TI } \rightarrow\end{array}$ & + & $0,162^{*}$ & 4,12 & Sí \\
$\begin{array}{l}\text { Reciprocidad } \\
\text { H7: H6: Propensión a innovar } \\
\text { en el ámbito de las TI } \rightarrow\end{array}$ & + & $0,253^{*}$ & 5,77 & Sí \\
\begin{tabular}{l} 
Apoyo recibido \\
\hline
\end{tabular}
\end{tabular}

Nota: *p<o,001; $\mathrm{t}(0,001 ; 4.999)=3,106644601$ (basado en $\mathrm{t}(4.999)$, una cola)

En este contexto de contrastación, si el usuario recibe apoyo de la comunidad se encuentra motivado a contribuir activamente y desea continuar la relación de la que disfruta. El usuario aporta además valor a otros miembros para mantener la reciprocidad mutuamente beneficiosa. El apoyo recibido influye en la integración social $(\beta=0,438 p<0,001)$ y la reciprocidad $(\beta=$ $0,585, p<0,001)$. Cuando una comunidad fomenta la reciprocidad de los miembros, estos desarrollan su integración social $(\beta=$ $0,322, p<0,001)$. Más aún, los usuarios con mayor PIIT se integran socialmente. La influencia sobre la integración es significativa ( $\beta$ $=0,188, p<0,001)$; su influencia sobre la sensación de sentirse apoyado $(\beta=0,253, p<0,001)$ y la reciprocidad $(\beta=0,162, p<$ $0,001)$ es tambien significativa.

Finalmente, para testar el efecto de la mediación de las hipótesis $\mathrm{H}_{4}$, se adopta el procedimiento de Preacher y Hayes (2008). El remuestreo bootstrap (5.000 submuestras) estimado del efecto indirecto $(0,189)$ es similar a la estimación puntual $(0,585$ $\mathrm{x} 0,322=0,188$; véase Figura 1). El valor o no se muestra en el intervalo de confianza (95 por ciento); se estima entre 0,147 y 0,234. La influencia del apoyo recibido sobre la integración social está significativamente mediada por la reciprocidad (véase Tabla 15).

Tabla 15. Efectos de mediación, hipótesis $\mathrm{H}_{4}$

\begin{tabular}{l|c|c|c|c}
\hline Relaciones & Media & Desvio & $\begin{array}{c}\text { Límite } \\
\text { inferior } \\
\text { IC }\end{array}$ & $\begin{array}{c}\text { Límite } \\
\text { superior } \\
\text { IC }\end{array}$ \\
\hline $\begin{array}{l}\text { H4: Apoyo recibido } \\
\rightarrow \text { Reciprocidad } \\
\rightarrow \text { Integración social }\end{array}$ & 0,188 & 0,128 & 0,147 & 0,234 \\
\hline
\end{tabular}


Figura 1. Modelo estructural (relaciones directas): Hipótesis y resultados

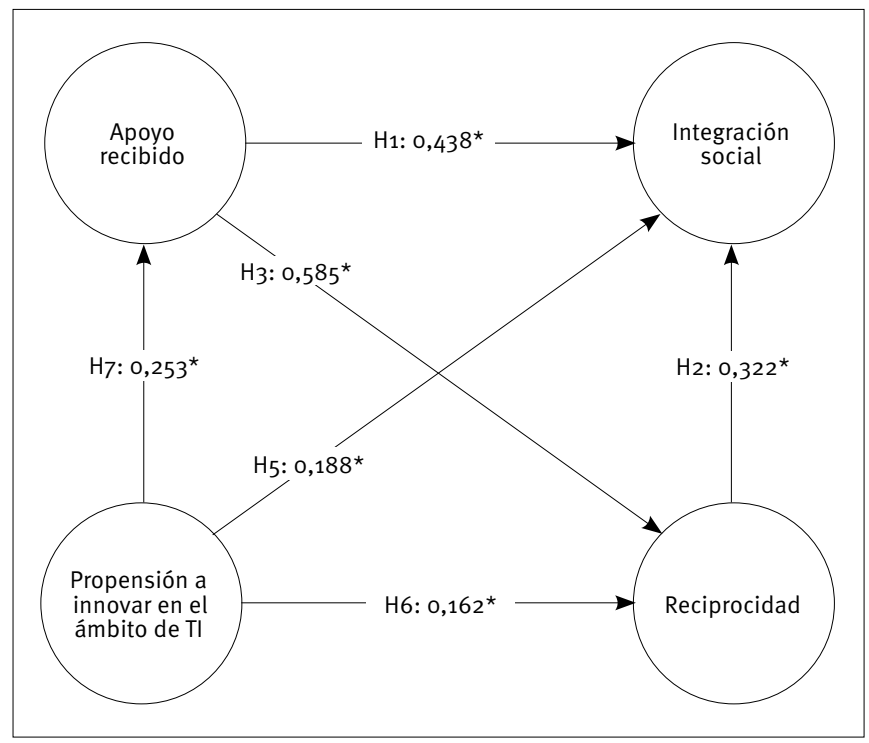

Nota: ${ }^{*} p<0,001 ; t(0,001 ; 4.999)=3,106644601$ [basado en $t(4.999)$, una cola]

\section{CONSIDERACIONES FINALES PARA LA DISCUSIÓN DE LOS RESULTADOS}

Estudios precedentes muestran que el apoyo que los miembros reciben, correlaciona positivamente con la participación de la comunidad (Sánchez-Franco \& Roldán-Salgueiro, 2010; Tonteri et al., 2011); tal y como se evidencia también en nuestro estudio. Un usuario receptor de apoyo en su comunidad, se beneficia de la misma, impulsándose su sentimiento de pertenencia. Nuestra investigación demuestra que el apoyo recibido influye positivamente en la integración social. No obstante, investigaciones precedentes se han centrado en las decisiones de aceptación sin valorar diferencias personales (Yi et al., 2006) o en el estudio de factores de la personalidad excesivamente amplios en sus consideraciones (Norman, 1963, Big Five Factors) - que no alcanzan resultados satisfactorios debido probablemente a los rasgos seleccionados (Hughes, Rowe, Batey, \& Lee, 2012). Factores de personalidad específicos o variables sociales podrían constituir mejores predictores de uso de las redes sociales (Krishnan \& Atkin, 2014; Wang, Jackson, Wang, \& Gaskin, 2015). Más aún, en numerosos estudios no se ofrecen propuestas explicativas de los efectos evidenciados (Ma \& Agarwall, 2007). En este sentido, y más allá de las influencias anteriormente descritas, nuestra propuesta de investigación ofrece conocimiento relevante y explicativo del papel de PIIT como diferencia individual estable y específica, y de la norma social de reciprocidad y sus influencias en el apoyo recibido y en la integración social.
En particular, nuestro estudio evidencia que el usuario que recibe apoyo aporta valor a otros miembros de la red y preserva el beneficio mutuo en el futuro; es decir, teje un sentimiento de reciprocidad. El apoyo recibido influye pues positivamente en la integración social y en la reciprocidad. La propia reciprocidad provoca además que los usuarios sean participantes y se identifiquen afectivamente con el grupo. Es probable que el individuo se sienta motivado para compartir experiencias y conocimientos cuanto más alto sea el nivel de expectativa de reciprocidad (Wasko \& Faraj, 2005), impulsándose a su vez los sentimientos de pertenencia e identidad (integración comunitaria). En definitiva, se fomenta la integración social. En suma, cabe concluir que la integración social mejora cuando el usuario percibe reciprocidad en su red social online.

Más aún, PIIT influye en la participación activa; tales usuarios consideran las redes sociales útiles y fáciles de usar, y desarrollan sentimientos de afiliación y apego. PIIT como rasgo estable de la personalidad genera beneficios colectivos debido al fomento de la cooperación y participación activa entre los miembros, y a su vez sentimientos de apoyo recibido, pertenencia e identidad social. Es decir, un mayor grado de PIIT incrementa la integración social y la sensación de apoyo recibido y con ello la participación duradera, como clave para el sostenimiento de la red social. Los usuarios propensos asumen el liderazgo y guían los comportamientos de otros mediante la retroalimentación de apoyo (Gurzick \& Lutters, 2009).

Finalmente, un usuario apoyado por la comunidad muestra mayor motivación para adoptar y practicar normas de reciprocidad. Le conduce a una mayor participación e integración con la comunidad. La influencia del apoyo recibido sobre la integración social está pues significativamente mediada por la reciprocidad.

En suma, nuestra investigación analiza el apoyo comunitario percibido, contribuyendo pues al debate sobre sus influencias en las sostenibilidad de las redes sociales. Por un lado, el apoyo recibido por los miembros influye en la integración social. Por otro, la reciprocidad impulsa la integración social (Kankanhalli et al., 2005). Los miembros que reciben contraprestaciones por sus aportaciones se sienten motivados a participar e integrarse socialmente. Asimismo, PIIT como rasgo estable del individuo se constituye como predictor del comportamiento de uso de las TI; i.e., una precondición escasamente analizada para explicar el uso sostenible de una red social (Sánchez-Franco et al., 2013). En suma, esta investigación ha puesto de manifiesto la importancia de la reciprocidad y PIIT para el desarrollo de investigaciones futuras y la planificación de estrategias eficaces por el gestor de la red. La red social es un grupo unido por intereses comunes susceptible de ser un grupo con una masa crítica de poder de 
decisión (Hagel \& Armstrong, 1997). Es esencial aprender a gestionar las redes de manera eficiente y activar las estrategias oportunas que incidan en la integración social.

\section{Implicaciones gerenciales}

En general, las redes sociales son fuente de reclamos, opiniones o sugerencias. Las organizaciones y marcas recaban información de los perfiles de los usuarios para su mejor conocimiento. Los usuarios también buscan información sobre las marcas (EMarketer, 2006) -permitiendo enlazarles con la web de e-commerce, y aprovechar la influencia de los usuarios líderes de opinión y de los contenidos virales (Gangadharbatla, 2009). Profundizar en los factores que influyen en el uso de la red social es pues una fuente de segmentación comercial y beneficios empresariales. Por otro lado, la mera participación del usuario no es condición suficiente del éxito de una red (Bhattacherjee, 2002); el éxito es consecuencia de la participación de los miembros y de los sentimientos de pertenencia e identidad social. La promoción de la integración social constituye pues un área de creciente relevancia.

En particular, existe un amplio debate relativo a los antecedentes de la participación del usuario y la integración comunitaria, y en torno a los efectos mediadores de los modelos propuestos en la literatura (Sánchez-Franco et al., 2012). La comprensión profunda de los motivos y diferencias individuales (Chou, 2010) que llevan a los individuos a integrarse socialmente con la comunidad es fundamental para las empresas. Por un lado, el apoyo recibido por los miembros influye en la integración social. Por otro, la reciprocidad impulsa la integración social (Yi et al., 2006). Asimismo, rasgos estables del individuo como PIIT constituyen predictores del comportamiento de uso regular de las TI; i.e. una precondición escasamente analizada para explicar el uso sostenible de una red social (Sánchez-Franco et al., 2013). Como manifiestan Arrasvuori y Olsson (2009), es esencial fomentar la participación y sociabilización online en el marco de la creación y mantenimiento de una relación. El incremento de relaciones entre miembros que comparten ideas e intereses comunes promueven conexiones profundas y ubicuas que mejoran la cohesión, fomentan el sentido de pertenencia y la participación (Bargh \& McKenna, 2004); desarrollan una mayor lealtad (Bagozzi \& Dholakia, 2006). La reciprocidad actúa como un código universal que impone la obligación de satisfacer los beneficios recibidos, provocando que las interacciones en la red aumenten. La visibilidad de las contribuciones enfatiza la reciprocidad, y consecuentemente la participación. Incluso miembros pasivos - mediante la observación de las normas de la comunidad - se familiarizan con la misma, impulsando su implicación y supervivencia (Wang \& Fesenmaier, 2004). El deseo y reconocimiento de estatus y prestigio incentivan a los usuarios a participar y contribuir (Hsu, Ju, Yen, \& Chang, 2007). El uso y establecimiento de símbolos comunes como el lenguaje compartido incrementa la comunalidad y dota a los usuarios de mayor confianza en sus interacciones (Yoo, Suh, \& Lee, 2001), fomentando la devolución de la ayuda recibida así como la percepción de sentimientos de identidad y pertenencia (Wasko \& Faraj, 2005). Es esencial establecer políticas de señalización para publicitar los privilegios de los usuarios participativos que señalice su reputación y suministre retroalimentación de su comportamiento (Tedjamulia, Dean, \& Albrecht, 2005) así como políticas sobre el lenguaje y normas que incrementen la confianza, la reciprocidad y el sentido de pertenencia. Si bien los usuarios pueden sentirse inicialmente inseguros en sus interacciones sociales, el sentido de pertenencia al grupo proporciona seguridad emocional (Kim, Lee, \& Hiemstra, 2004) y lealtad a la comunidad (McMillan, 1996); enfatiza la autorrevelación de información intima que ayuda a los responsables de la comunidad a conocer mejor a sus miembros y establecer criterios de segmentación.

Más aún, la acceso pertinente de los usuarios a la información fomenta la participación para el éxito sostenido de la red y constituye un motivo para que los usuarios se integren (Phang, Kankanhalli, \& Sabherwal, 2009). Diversos estudios destacan la importancia de la usabilidad, la calidad y pertinencia de la información, la relevancia gráfica y visual, la política de incentivos y apoyo mutuo, entre otros, y también su influencia en la sensación de disfrute y sentido de pertenencia (Lu, Phang, \& Yu, 2011; Wasko \& Faraj, 2005). En particular, la usabilidad provoca que las interacciones sean sencillas y manejables; el sistema es útil y favorece la disposición de los usuarios a colaborar. El gestor debe establecer políticas que prevengan los fallos de usabilidad y faciliten la navegación y acceso a información pertinente, por ejemplo, a través de la personalización que incremente el control percibido.

Por último, el gestor debe valorar el grado de PIIT de los miembros. Los individuos con menor propensión infravaloran la utilidad y facilidad de uso, asumen menos riesgos y consecuentemente menores expectativas de reciprocidad y mayores dificultades de integración. El gestor debe fomentar políticas de actividades online con distintos niveles de complejidad donde estos usuarios participen de una forma básica y gradual a través del refuerzo positivo y desarrollen su integración social. El gestor debe también estimular a los usuarios con mayor PIIT fomentando la exploración de utilidades avanzadas (Chou, 2010) y su reconocimiento por la comunidad (Lu et al., 2011). El gestor debe mantener relaciones afectivas con estos 
usuarios e incentivar su papel como líderes mediante políticas de recompensas que estimulen su creatividad, otorgándoles privilegios premium a diversas funcionalidades de la red. PIIT es pues esencial para identificar los roles de los usuarios en su aceptación (Agarwal \& Prasad, 1998).

En suma, nuestro trabajo sugiere a los gestores de las comunidades online estrategias y acciones relevantes para potenciar PIIT y la reciprocidad con objeto de fomentar la integración social y segmentar sus comunidades impulsando así el éxito de la red social online y el potencial beneficio empresarial derivado de la misma.

\section{Limitaciones para consideraciones finales}

De la interpretación de los resultados del estudio se desprenden limitaciones y oportunidades para futuras investigaciones. En primer lugar, las escalas de medidas propuestas evalúan las intenciones de los consumidores. Es necesario, no obstante, investigar también el comportamiento real de creación y mantenimiento de la integración social. En segundo lugar, esta investigación no incluye todas las variables relevantes, e.g., las habilidades del usuario, orientación sexual, tradiciones, educación y rasgos como la humildad, la empatía, la propensión a confiar y la propensión a desempeñar conductas prosociales. Características moderadoras de la personalidad como la timidez, el narcisismo o la autoestima (Barker, 2009; Steinfield, Ellison, \& Lampe, 2008) también muestran relaciones con las motivaciones de uso. Asimismo, las características, el objetivo y las normas de cada red social evidencian su relación con el comportamiento (Pappacharissi, 2009). En tercer lugar, estudios previos manifiestan la existencia de distintas propensiones en el uso de las diferentes redes sociales encontrando diferencias significativas entre nacionalidades y razas (Hargittai, 2007). El sexo también propicia usos diversos de las redes sociales (Lenhart, Madden, Macgill, \& Smith, 2007), afectando al modo en que los jóvenes interactúan y se comportan y a su participación en redes sociales (Agosto \& Abbas, 2010). En cuarto lugar, el carácter no probabilístico del estudio cuestiona la generalización de los resultados. Aunque el modelo propuesto es testado en una muestra superior a 490 individuos, es difícil generalizar debido a la selección no aleatoria de los participantes. Por último, el diseño transversal del estudio impide inferencias inequívocas de causalidad. Es necesario realizar un estudio longitudinal que incluya la variable temporal y relaciones causales entre las variables estudiadas así como un análisis transversal entre redes generalistas vs temáticas.

\section{FUTURAS LÍNEAS DE INVESTIGACIÓN}

En primer lugar, el uso de estrategias de moderación en las comunidades promueven la participación de los miembros y atraen a nuevos usuarios (Berge, 1992; Collins \& Berge, 1997). El liderazgo y la moderación natural que ejercen diversos usuarios como organizadores comunitarios y guías de contenidos son aspectos relevantes. Las contribuciones son más consistentes, aportan mayor seguridad al resto de usuarios y adhesión a la norma de reciprocidad, incrementan los sentimientos de identificación, apoyo mutuo, e impulsan la participación (Koh, Kim, Butler, \& Bock, 2007). La investigación debe profundizar pues en los efectos de moderación sobre la integración social y la existencia de diferencias según el tipo de red social, PIIT, liderazgo, y reciprocidad.

Investigaciones previas señalan que la forma de uso y los beneficios de las redes son influidos por las diferencias individuales en personalidad o habilidades de los usuarios (Anderson, Fagan, Woodnutt, \& Chamorro-Premuzic, 2012). La homogeneidad en las características psicográficas de los miembros conduce precisamente a un mayor interés, participación y apego. Investigaciones futuras deben pues profundizar en el conocimiento de la integración social y su influencia según el sexo, edad o nivel de estudios (Gurzick, Ozok, \& Morris, 2007; Lee, Fairhurst, \& Cho, 2013). Es esencial además profundizar en el estudio de rasgos psicográficos que permitan que el usuario se identifique con su grupo, potenciando el sentimiento de pertenencia y participación. Por ejemplo, diferencias culturales afectan el grado en que los individuos valoran la reciprocidad (Shore \& Coyle-Shapiro, 2003). En suma, es necesario diseñar estudios longitudinales sobre el ciclo de vida que experimenta la participación activa así como los efectos de la personalidad del individuo en la consideración de la reciprocidad y su repercusión en la integración social.

En segundo lugar, la usabilidad promueve la creencia de habilidad con el sistema y genera una sensación de autoeficacia. La usabilidad podrías ser pues un impulsor de la reciprocidad y la interacción social. Más aún, el uso de un lenguaje compartido y la utilidad, exactitud y exhaustividad del contenido promueven la reciprocidad del usuario, y fomentan la integración social y la lealtad. Futuros estudios deben analizar las habilidades del usuario, la usabilidad, sexo y género del miembro, orientación sexual, o su edad, junto a la implicación o familiaridad.

En tercer lugar, las relaciones son una combinación de conexiones online y offline (Gurzick \& Lutters, 2009). El análisis de los encuentros fuera de la red permite complementar adecuadamente la formación de la integración social. 
Finalmente, con el incremento del uso del teléfono móvil, tabletas y otros dispositivos los usuarios permanecen conectados a la red de cualquier forma, lugar y momento del día. La viabilidad no solo se basa en la lealtad afectiva de sus usuarios sino también en la promoción del uso y participación ubicua en los servicios asociados a la red. Es relevante analizar cómo afecta el momento, forma y lugar de conexión en la creación y mantenimiento de la integración.

\section{REFERENCIAS}

Agarwal, R., \& Prasad, J. A. (1998). A conceptual and operational definition of personal innovatiness in the domain of information technology. Information System Research, 9(2), 204-215. doi:10.1287/ isre.9.2.204

Agosto, D., \& Abbas, J. (2010). High school seniors' social network and other ICT use preferences and concerns. Proceedings of the American Society for Information Science and Technology, 47(1), 1-10. doi:10.1002/meet.14504701025

Ahn, J. (2011). The effects of social network sites on adolescents' social and academic development: Current theories and controversies. Journal on the American Society for Information Science and Technology, 62(8), 1435-1445. doi:10.1002/asi.21540

Ahn, J. (2012). Teenagers' experiences with social network sites: Relationships to bridging and bonding social capital. The Information Society: An International Journal, 28(2), 99-109. doi:10.1080/019722 43.2011.649394

Algesheimer, R., Dholakia, U. M., \& Herrmann, A. (2005). The social influence of brand community: Evidence from European car clubs. Journal of Marketing, 69(3), 19-34.

Anderson, B., Fagan, P., Woodnutt, T., \& Chamorro-Premuzic, T. (2012). Facebook psychology: Popular questions answered by research. Psychology of Popular Media Culture, 1(1), 23-37.

Arnett, J. J. (2004). Emerging adulthood: The winding road from the late teens through the twenties. New York, EUA: Oxford University Press.

Arrasvuori, J., \& Olsson, T. (2009). A model for analyzing online communities. International Journal of Business and Information, 4(2), $115-136$.

Bagozzi, R. P., \& Dholakia, U. M. (2006). Antecedents and purchase consequences of customer participation in small group brand communities. International Journal of Research in Marketing, 23(1), 45- 61. doi:10.1016/j.jiresmar.2006.01.005

Barclay, D. W., Thompson, R., \& Higgins, C. (1995). The partial least squares (PLS) approach to causal modeling: Personal computer adoption and use an illustration. Technology Studies, 2(2), 285-309.

Bargh, J. A., \& McKenna, K. Y. A. (2004). The internet and social life. Annual Review of Psychology, 55, 573-590. doi:10.1146/annurev. psych.55.090902.141922

Barker, V. (2009). Older adolescents' motivations for social network site use: The influence of gender, group identity, and collective self esteem. CyberPsychology \& Behavior, 12(2), 209-213. doi:10.1089/ cpb.2008.0228
Berge, Z. L. (1992). The role of the moderator in a scholarly discussion group (SDG). Recuperado de http://www.researchgate.net/ publication/238671678_The_role_of_the_moderator_in_a_ scholarly_discussion_group_(sdg).

Bhattacherjee, A. (2002). Individual trust in online firm: Scale development and initial test. Journal of Management Information Systems, 19(1), 211-241. doi:10.1080/07421222.2002.11045715

Blanchard, A. L. (2008). Testing a model of sense of virtual community. Computers in Human Behavior, 24(5), 2107-2123. doi:10.1016/j. chb.2007.10.002

Boneva, B. S., Quinn, A., Kraut, R. E., Kiesler, S., \& Shklovski, I. (2006). Teenage communication in the instant messaging era. In R. Kraut, M. Brynin, \& S. Kiesler (Eds.). Information technology at home (pp. 612672). New York: Oxford University Press.

Brodie, R. J., Ilic, A., Juric, B., \& Hollebeek, L. (2013). Consumerengagement in a virtual brand community: An exploratory analysis. Journal of Business, 66(1), 105-114. doi:10.1016/j.jbusres.2011.07.029

Calder, B. J., Lynn, W. P., \& Tybout, A. M. (1981). Designing research for application. Journal of Consumer Research, 8(2), 197-207.

Casaló, L. V., Flavián, C., \& Guinalíu, M. (2007). Antecedents and effects of participation in virtual brand communities. I.4DIS International Conference on IYEB Based Communities, Salamanca, España.

Casaló, L. V., Flavián, C., \& Guinalíu, M. (2011). Observación y participación en comunidades virtuales comerciales: El caso del sector del turismo. REIM-Revista Española de Investigación de Marketing, 15(2), 113-136.

Chin, W. W. (1998a). Issues and opinion on structural equation modeling. MIS Quarterly, 22(1), 7-16.

Chin, W. W. (1998b). The Partial Least Squares approach for structural equation modeling. In: G. A. Marcoulides (Ed.). Modern methods for business research (pp. 295-336). Mahwah, NJ, EUA: Lawrence Erlbaum Associates.

Chin, W. W. (2000). Frequently asked questions: Partial Least Squares \& PLS-Graph. Recuperado de http://disc-nt.cba.uh.edu/chin/plsfaq.htm.

Chou, S. H. W. (2010). Why do members contribute knowledge to online communities? Online Information Review, 34(6), 829-854. doi:10.1108/14684521011099360

Cohen, J. (1988). Statistical power analysis for the behavioral sciences. Hillsdale, NJ, EUA: Lawrence Erlbaum.

Collins, M. P., \& Berge, Z. L. (1997). Moderating online electronic discussion groups. American Educational Research Association, Chicago, IL, EEUU.

Cothrel, J., \& Williams, R. (1999). Online communities: Getting the most out of online discussion and collaboration. Knowledge Management Review, 1(6), 20-25.

EMarketer. (2006). Social networking online boosts bottom line. Recuperado de http://www.emarketer.com.

Falk, R. F., \& Miller, N. B. (1992). A primer for soft modeling. Akron, Ohio, EUA: The University of Akron.

Fornell, C., \& Larcker, D. (1981). Evaluating structural equation models with unobservable variables and measurement error. Journal of Marketing Research, 18(1), 39-50. doi:10.2307/3151312

Gangadharbatla, H. (2009). Individual differences in social networking site adoption. In C. R. Livermore \& K. Setzekorn (Eds), Social Networking Communities and E-Dating Services: Concepts and Implications (pp. 1-17). Hershey, PA: IGI Global. 
García, M. C., Alonso, J., \& Hoyo, M. (2013). La participación de los jóvenes en las redes sociales: Finalidad, oportunidades y gratificaciones. Anàlisi: Quaderns de Comunicacióicultura, (48), 95-110.

Gefen, D. (2000). E-commerce: The role of familiarity and trust. Omega, 28(6), 725-737. doi:10.1016/S0305-0483(00)00021-9

Geisser, S. (1975). The predictive sample reuses method with applications. Journal of the American Statistical Association, 70(350), 320-328. doi:10.2307/2285815

Gómez, M., Roses, S., \& Farias, P. (2012). El uso académico de las redes sociales en universitarios. Revista Científica de Educomunicación Comunicar, 19(38), 131-138. doi:10.3916/c38-2012-03-04

Gonnet, J. P. (2010). Reciprocidad, interacción y doble contingencia: Una aproximación a lo social. A Parte Rei, (71), 1-10.

Gracia, E., García, F., \& Musitu, G. (1995). Macrosocial determinants of social integration: Social class and area effect. Journal of Community \& Applied Social Psychology, 5(2), 105-119. doi:10.1002/ casp. 2450050204

Gurzick, D., \& Lutters, W. G. (2009). Towards a design theory for online communities. 4th International Conference on Design Science Research in Information Systems and Technology, Article $n$. 11, ACM, New York, NY, USA.

Gurzick, D., Ozok, A. A., \& Morris, B. (2007). Online communication \& baby boomers: Current use, preferences and future expectations. Americas Conference on Information Systems (AMCIS), Keystone, CO, EUA.

Hagel, J., \& Armstrong, A. (1997). Net gain: Expanding markets through virtual communities. Boston, EUA: Harvard Business School Press.

Hargittai, E. (2007). Whose space? Differences among users and non-users of social network sites. Journal of ComputerMediated Communication, 13(1), 276-297. doi:10.1111/j.10836101.2007.00396.x

Harris, L., \& Dennis, C. (2011). Engaging customers on facebook: Challenges for e-retailers. Journal of Consumer Behaviour, 10(6), 338346. doi:10.1002/cb.375

Herrero, J., \& Gracia, E. (2007). Measuring perceived community support: Factorial structure, longitudinal invariance, and predictive validity of the PCSQ (Perceived Community Support Questionnaire). Journal of Community Psychology, 35(2), 197-217. doi:10.1002/jcop.20143

Hester, A. J. (2011). A comparative analysis of the usage and infusion of wiki and non-wiki-based knowledge management systems. Information Technology and Management, 12(4), 335-355. doi:10.1007/s10799-010-0079-9

Hsu, M. H., Ju, T., Yen, C. H., \& Chang, C. M. (2007). Knowledge sharing behavior in virtual communities: The relationship between trust, selfefficacy, and outcome expectations. International Journal of HumanComputer Studies, 65(2), 153-169. doi:10.1016/j.ijhcs.2006.09.003

Hughes, D. J., Rowe, M., Batey, M., \& Lee, A. (2012). A tale of two sites: Twitter vs. Facebook and the personality predictors of social media usage. Computers in Human Behavior, 28(2), 561-569. doi:10.1016/j. chb.2011.11.001

Instituto Nacional de Estadistica. (2014). Encuesta sobre equipamiento y uso de tecnologías de información y comunicación en los hogares. Nota de prensa, 8. Recuperado de www.ine.es/prensa/prensa.htm.

Interactive Advertising Bureau. (2015, Enero). VI Estudio Redes Sociales de IAB Spain Versión Abierta. Recuperado de http://www.iabspain. net/wp-content/uploads/downloads/2015/01/Estudio_Anual_ Redes_Sociales_2015.pdf.
Ito, M., Baumer, S., Bittanti, M., Boyd, D., Cody, R., \& Herr-Stephenson, B. (2009). Hanging out, messing around, and geeking out: Kids living and learning with new media. Cambridge, MA, EUA: MIT Press.

Kankanhalli, A., Tan, B. C. Y., \& Wei, K. K. (2005). Contributing knowledge to electronic knowledge repositories: An empirical investigation. MIS Quarterly, 29(1), 113-143

Kim, W. G., Lee, C., \& Hiemstra, S. J. (2004). Effects of an online virtual community on customer loyalty and travel product purchases. Tourism Management, 25(3), 343-355. doi:10.1016/So261-5177(03)00142-0

Koh, J., Kim, Y. G., Butler, B., \& Bock, G. W. (2007). Encouraging participation in virtual communities. Communications of the ACM, 5o(2), 68-73. doi:10.1145/1216016.1216023

Krishnan, A., \& Atkin, D. (2014). Individual differences in social networking site users: The interplay between antecedents and consequential effect on level of activity. Computers in Human Behavior, 40, 111-118. doi:10.1016/j.chb.2014.07.045

LaRose, R., Eastin, M. S., \& Gregg, J. (2001). Reformulating the internet paradox: Social cognitive explanations of internet use and depression. Journal of Online Behavior, 1(2).

Lee, H. J., Fairhurst, A., \& Cho, H. J. (2013). Gender differences in consumer evaluations of service quality: Self-service kiosks in retail. The Service Industries Journal, 33(2), 248-265. doi:10.1080/026420 69.2011 .614346

Lee, Y., Kozar, K. A., \& Larsen, K. R. T. (2003). The technology acceptance model: Past, present, and future. Communications of the Association for Information Systems, 12 (50), 751-780.

Lenhart, A., Madden, M., Macgill, A. R., \& Smith, A. (2007). Teens and social media. Washington, DC, EEUU: Pew Internet \& American Life Project.

Leung, L., \& Lee, P. S. N. (2012). The influences of information literacy, internet addiction and parenting styles on internet risks. New Media \& Society, 14(1), 117-136. doi:10.1177/1461444811410406

Lu, X., Phang, C. W., \& Yu, J. (2011). Encouraging participation in virtual communities through usability and sociability development: An empirical investigation. ACM SIGMIS Database, 42(3), 96-114.

Ma, M., \& Agarwal, R. (2007). Through a glass darkly: Information technology design, identity verification, and knowledge contribution in online community. Information Systems Research, 18(1), 4267. doi:10.1287/isre.1070.0113

McKnight, D. H., Kacmar, C. J., \& Choudhury, V. (2004). Dispositional trust and distrust distinctions in predicting high - and low-risk internet expert advice site perceptions. e-Service Journal, 3(2), 35-58. doi:10.2979/esj.2004.3.2.35

McMillan, D. W. (1996). Sense of community. Journal of Community Psychology, 24(4), 315-325. doi:10.1002/(sici)15206629(199610)24:4‘315::aid-jcop2〉3.0.c0;2-t

Nahapiet, J., \& Ghoshal, S. (1998). Social capital, intellectual capital, and the organizational advantage. Academy of Management Review, 23(2), 242-266.

Norman, W. T. (1963). Toward an adequate taxonomy of personality attributes: Replicated factor structure in peer nomination personality ratings. Journal of Abnormal and Social Psychology, 66(6), 574-583. doi:10.1037/hoo40291

Nowell, B., \& Boyd, N. (2010). Viewing community as responsibility as well as resource: Deconstructing the theoretical roots of psychological sense of community. Journal of Community Psychology, 38(7), 828 841. doi:10.1002/jcop.20398 
Nunnally, J. (1978). Psychometric theory (2nd ed.). New York, EUA McGraw-Hill.

Online Business School. (2014). España aumenta el número de usuarios activos en redes sociales en 2014 y llega a los 17 millones. Recuperado de http://www.obs-edu.com/noticias/estudio-obs/ espana-aumenta-el-numero-de-usuarios-activos-en-redes-socialesen-2014-y-llega-los-17-millones/.

Osborne, J., \& Overbay, A. (2008). Best practices in data cleaning: How outliers and "fringeliers" can increase error rates and decrease the quality and precision of your results. In J. W. Osborne (Ed.). Best practices quantitative methods (pp. 205-213). Thousand Oaks, CA, EUA: SAGE Publications.

Osorio, A. B. (2009). Perfil y motivaciones del voluntariado juvenil de mayores (Tesis de doctorado, Universidad de Granada, España).

Osorio, M. J., Molero, M. M., Pérez, M. C., \& Mercader, I. (2014). Redes sociales en internet y consecuencias de su uso en estudiantes universitarios. International Journal of Developmental and Educational Psychology INFAD Revista de Psicología, 3(1), 585-592.

Pappacharissi, Z. (2009). The virtual geographies of social networks: A comparative analysis of Facebook, Linkedin, and ASmallWorld. New Media \& Society, 11(1), 199-220. doi:10.1177/1461444808099577

Phang, C., Sutanto, J., Kankanhalli, A., Li, Y., Tan, B., \& Teo, H. (2006). Senior citizens' acceptance of information systems: A study in the context of e-government services. IEEE Transactions on Engineering Management, 53(4), 555-569. doi:10.1109/tem.2006.883710

Phang, C. W., Kankanhalli, A., \& Sabherwal, R. (2009). Usability and sociability in online communities: A comparative study of knowledge seeking and contribution. Journal of the Association for Information Systems, 10(10), 721-747.

Preacher, K. J., \& Hayes, A. F. (2008). Asymptotic and resampling strategies for assessing and comparing indirect effects in multiple mediator models. Behavior Research Methods, 40(3), 879-891. doi:10.3758/brm.40.3.879

Preece, J. (2000). Online communities: Designing usability, supporting sociability. Chichester, Reino Unido: Wiley.

Putnam, R. (1995). Tuning in, tuning out: The strange disappearance of social capital in America. Political Science \& Politics, 28(4), 664-683. doi:10.2307/420517

Radmacher, K., \& Azmitia, M. (2006). Are there gendered pathways to intimacy in early adolescents' and emerging adults' friendships? Journal of Adolescent Research, 21(4), 415-448. doi:10.1177/0743558406287402

Rogers, E. M. (1995). Diffusion of innovations (4th ed.). New York, EUA: Free Press.

Rogers, E. M. (2003). Diffusion of innovations (5th ed.). New York, EUA: Free Press.

Rothaermel, F. T., \& Sugyama, S. (2001). Virtual internet communities and commercial success: Individual and community-level theory grounded in the atypical case of TimeZone.com. Journal of Management, 27(3), 297-312. doi:10.1177/014920630102700305

Ruiz, R. (2005). Estudio e intervención en la conducta prosocial-altruista (Tesis de doctorado, Universidad de Córdoba, España).

Ruiz, J., Izquierdo, M., \& Piñera, T. (1998). Aportaciones en torno a los usuarios en documentación. Documentación de las Ciencias de la Información, (21), 11-25.

Sánchez-Franco, M. J., Buitrago-Esquinas, E. M., \& Hernández-Mogollón, J. M. (2012). Antecedentes sociales y psicológicos del compromiso comunitario: Un análisis del comportamiento del usuario de una red social de relaciones. Cuadernos de Economía y Dirección de la Empresa, 15(4), 205-220. doi:10.1016/j.cede.2012.04.007

Sánchez-Franco, M. J., Buitrago-Esquinas, E. M., \& Yñiguez-Ovando, R. (2013). Personal traits, social capital and integration in the domain of social network sites. 2nd Global Innovation and Knowledge Academy (GIKA), Universidad Politécnica de Valencia, España.

Sanchez-Franco, M. J., \& Roldan-Salgueiro, J. L. (2010). Expressive aesthetics to ease perceived community support: Exploring personal innovativeness and routinized behaviour as moderators in Tuenti. Computers in Human Behavior, 26(6), 1445-1457. doi:10.1016/j. chb.2010.04.023

Shore, L. M., \& Coyle-Shapiro, J. A. M. (2003). New developments in the employee-organization relationship. Journal of Organizational Behavior, 24(5), 443-450. doi:10.1002/job.212

Steinfield, C., Ellison, N. B., \& Lampe, C. (2008). Social capital, selfesteem, and use of online social network sites: A longitudinal analysis. Journal of Applied Developmental Psychology, 29(6), 434445. doi:10.1016/j.appdev.2008.07.002

Subrahmanyama, K., Reichc, S. M., Waechterb, N., \& Espinozab, G. (2008). Online and offline social networks: Use of social networking sites by emerging adults. Social Networking on the Internet, 29(6), 420-433. doi:10.1016/j.appdev.2008.07.003

Sue, W. (2012). The impact of social networking sites on college students' consumption patterns (Tesis doctoral, Marshall University, EEUU).

Tedjamulia, S. J. J., Dean, D. L., \& Albrecht, C. C. (2005). Motivating content contributions to online communities: Toward a more comprehensive theory. 38th Annual Hawaii International Conference on System Sciences, Hawaii, EEUU.

Thatcher, J. B., \& Perrewé, P. L. (2002). An empirical examination of individual traits as antecedents to computer anxiety and computer self-efficacy. MIS Quarterly, 26(4), 381-396. doi:10.2307/4132314

Tonteri, L., Kosonen, M., Ellonen, H. K., \& Tarkiainen, A. (2011). Antecedents of an experienced sense of virtual community. Computers in Human Behavior, 27(6), 2215-2223. doi:10.1016/j.chb.2011.06.018

Vallor, S. (2012). Flourishing on Facebook: Virtue friendship \& new social media. Ethics and Information Technoly, 14(3), 185-199. doi:10.1007/ s10676-010-9262-2

Wang, J. L., Jackson, L. A., Wang, H. Z., \& Gaskin, J. (2015). Predicting social networking site (SNS) use: Personality, attitudes, motivation and internet self-efficacy. Personality and Individual Differences, 80, 119-124. doi:10.1016/j.paid.2015.02.016

Wang, Y., \& Fesenmaier, D. R. (2003). Assessing motivation of contribution in online communities: An empirical investigation of an online travel community. Electronic Markets, 13(1), 33-45. doi:10.1080/1019678032000052934

Wang, Y., \& Fesenmaier, D. R. (2004). Towards understanding members' general participation in and active contribution to an online travel community. Tourism Management, 25(6), 709-722. doi:10.1016/j. tourman.2003.09.011 
Wasko, M. M., \& Faraj, S. (2005). Why should I share? Examining social capital and knowledge contribution in electronic networks of practice. MIS Quarterly, 29(1), 35-57.

Wethington, E., \& Kessler, R. C. (1986). Perceived support, received support, and adjustment to stressful life events. Journal of Health and Social Behavior, 27(1), 78-89.

Wiertz, C., \& Ruyter, K. de. (2007). Beyond the call of duty: Why consumers contribute to finn-hosted commercial online communities. Organization Studies, 28(3), 347-376. doi:10.1177/0170840607076003
Woszczynski, A. B., Roth, P. L., \& Segars, A. H. (2002). Exploring the theorical foundations of playfulness in computer interactions. Computers in Human Behavior, 18(4), 369-388. doi:10.1016/S07475632(01)00058-9

Yi, M. Y., Fiedler, K. D., \& Park, J. S. (2006). Understanding the role of individual innovativeness in the acceptance of IT-based innovations: Comparative analyses of models and measures. Decision Sciences, 37(3), 393-426. doi:10.1111/j.1540-5414.2006.00132.x

Yoo, W. S., Suh, K. S., \& Lee, M. B. (2001). Exploring the factors enhancing member participation in virtual communities. Pacific Asia Conference on Information Systems (PACIS 2001) Proceedings. Paper 38 